\title{
Parental Involvement, Health Behaviors and Mental Health among School-going Adolescents in Six Asian Countries
}

\author{
Supa Pengpid ${ }^{1,2}$ and Karl Peltzer ${ }^{2,3^{*}}$ \\ ${ }^{I}$ ASEAN Institute for Health Development, Mahidol University, Salaya, Nakhonpathom 73170 , \\ Thailand \\ ${ }^{2}$ Department of Research Development and Innovation, University of Limpopo, Sovenga 0727, \\ South Africa \\ ${ }^{3}$ HIVIAIDS/STIsland TB (HAST), Human Sciences Research Council, Private Bag X41, \\ Pretoria 0001, South Africa \\ *Corresponding author. E-mail: kpeltzer@hsrc.ac.za \\ https://doi.org/10.12982/CMUJASR.2016.0007
}

ABSTRACT
parental involvement, health behaviors, and mental health among adolescents in six Asian countries (Iraq, Kuwait, Malaysia, Mongolia, Philippines, and Vietnam). Using data from the cross-sectional 'Global School-Based Student Health Survey (GSHS)', 44,231 adolescents (mean age 15.1 years, SD=1.5) were assessed with a self-administered structured questionnaire. In multivariable regression, higher levels of parental involvement were positively associated with fruit and vegetable consumption and physical activity in half of the study countries, and negatively associated with bullying victimization, having been in a physical fight, loneliness, anxiety, and suicidal ideation in most countries. Parental involvement and/or support may be effective in increasing the number of positive health behaviors, including fruit and vegetable consumption, physical activity, no violent behaviors, school attendance, and mental health in this adolescent population.

Keywords: Parental involvement, Health risk behavior, Mental health, Adolescents, Asia 


\section{INTRODUCTION}

"Previous research has provided considerable support for the idea that increased parental support and control are strong determinants of lower prevalence levels of adolescent risk behavior" (de Looze et al., 2012). According to Newman et al. (2008),

There are many dimensions of the adolescent-parent relationship that might influence adolescent health and developmental outcomes, as well as the development of risky health behaviors. Specific parenting behaviors that have been found to influence adolescent health and risky health behaviors include type of discipline (consistent versus inconsistent), level of parental involvement, level of parental monitoring, type of communication, and parenting style.

Most research on parental involvement has been conducted on individual risk behaviorsin high-income countries, and there is a need to investigate a broad range of health risk behaviors, such as dietary behavior, physical activity, violence, truancy, and mental health, in developing countries, including Asia (Newman et al., 2008).

\section{Dietary behavior and physical activity}

In a study among adolescents in the USA, "maternal, paternal, and peer support for healthy eating were positively associated with the vegetable and fruit pattern" (Cutler et al., 2011). Lack of parental involvement in a study among adolescents in California associated with low fruit and vegetable consumption (Mistry et al., 2009), and adolescents who reported to have authoritative parents ate more fruit per day than those who described parents as neglectful (Pearson et al., 2010). Parental support was positively associated with physical activity among adolescents in California (Mistry et al., 2009), Estonia (Raudsepp, 2006), Pakistan (Ahmed et al., 2016), as well as in a systematic review (Yao and Rhodes, 2015). In a national representative survey among adolescents in the USA, "children's perception of parental support and parent's reports of direct support were strongly related to organized physical activity" (Heitzler et al., 2006).

\section{Violence and school truancy}

In a study among adolescents in Turkey, poor paternal support monitoring was associated with both bullying victimization and perpetration (Erginoz et al., 2015). Among school-going adolescents, parental supervision protected children from physical fighting in the Philippines (Rudatsikira et al., 2008), from being bullied in China (Hazemba et al., 2008) and from school truancy in Swaziland and Zambia (Siziya et al., 2007; \& Muula et al., 2012). In a meta review, parental monitoring was found to be protective against delinquency (Hoeve et al., 2009).

\section{Mental health}

Parental regulation and monitoring has been associated with lower levels 
of suicidal ideation and depression (Barber, 2002). Arat and Wong (2016) found that parental bonding protected adolescents from loneliness in four out of six African countries, and parental connectedness was negatively associated with anxiety, depression, and suicidal ideation in three countries. In several countries, parental supervision was positively associated with mental distress indicators (loneliness, anxiety, and suicidal ideation) (Arat \& Wong, 2016). Among adolescents, parental involvement was associated with better mental health in India (Hasumi et al., 2012), with fewer symptoms of depression and suicidal thoughts in Thailand (Tammariello et al., 2012), and with fewer mental health issues in the Caribbean (Abdirahman et al., 2012). In a study in Malaysia, mother and father closeness was negatively associated with suicidal ideation among adolescents (Wu \& Yaacob, 2017). In a meta review of intervention trials, enhanced parental involvement prevented internalizing problems, such as anxiety, in adolescents (Yap et al., 2016).

The aim of this investigation was to assess the association between parental involvement (support and monitoring) and health behaviors and mental health among adolescents in six Asian countries.

\section{METHODOLOGY}

\section{Participants and procedures}

This study was a secondary analysis of already existing data from the 'Global School-based Health Survey'
(GSHS) from six Asian countries (all those that had utilized the modules related to this study). Details about the GSHS and its data can be accessed. The GSHS used a 'two-stage (schools and classrooms) cluster sampling design to generate nationally representative samples of students in years 1 to 4 in secondary schools' (CDC, 2017). "Students completed a self-administered questionnaire under the supervision of trained survey administrators" (CDC, 2017). National Ethics Committees approved the study protocol, and informed consent was obtained from the students, parents and/or school officials (CDC, 2017).

\section{Measures}

This study utilized the GSHS questionnaire's modules on diet, physical activity, violence, injury, mental health, and other health related behaviors, along with demographics (CDC, 2017). Adequate fruit consumption was defined as two or more servings a day and adequate vegetable consumption as three or more servings a day (CDC, 2013). Adequate physical activity was defined as 'at least 60 minutes of moderate to vigorous-intensity physical activity daily' (WHO, 2010). The study variables are described in Table 1. 


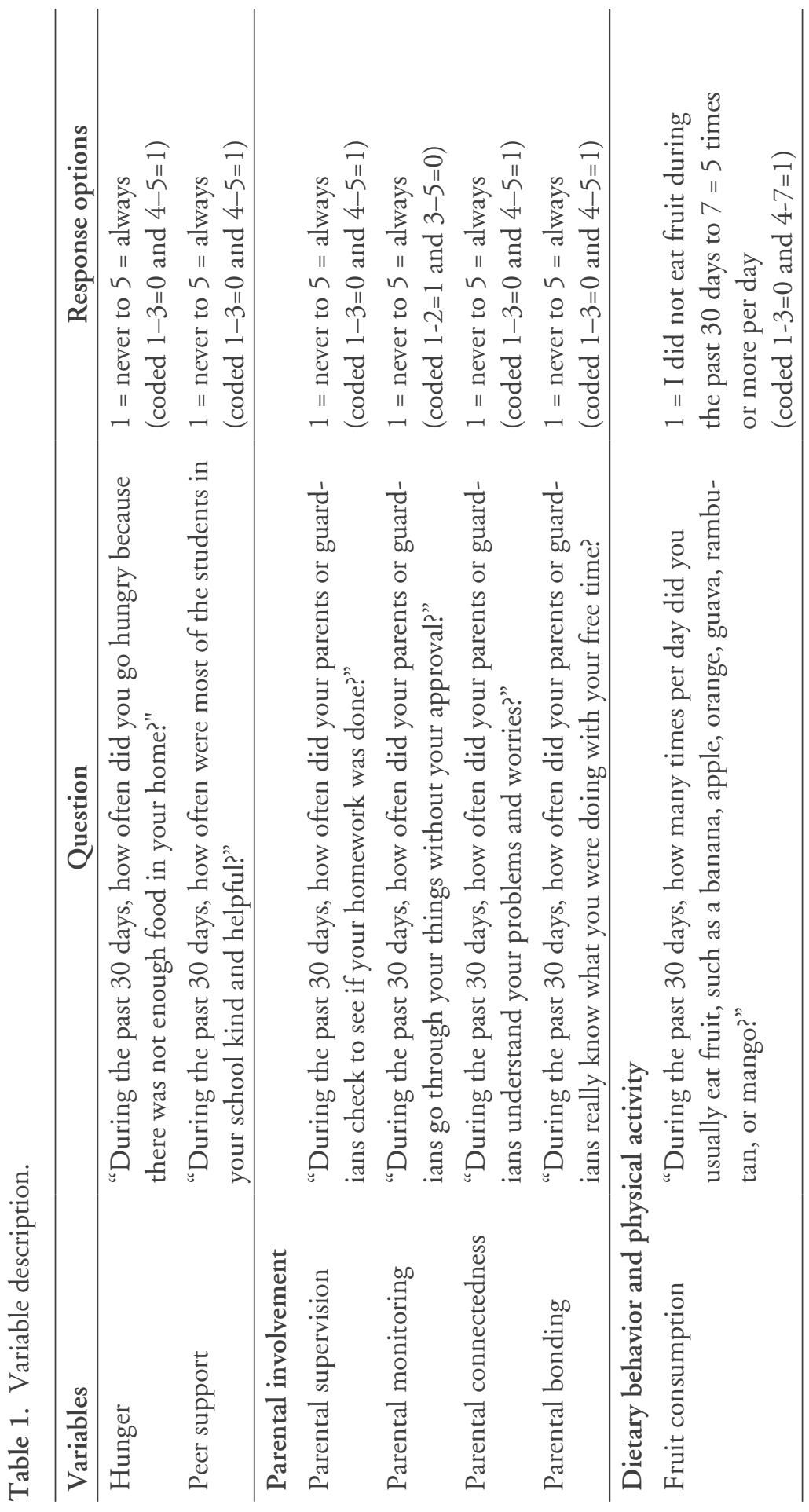




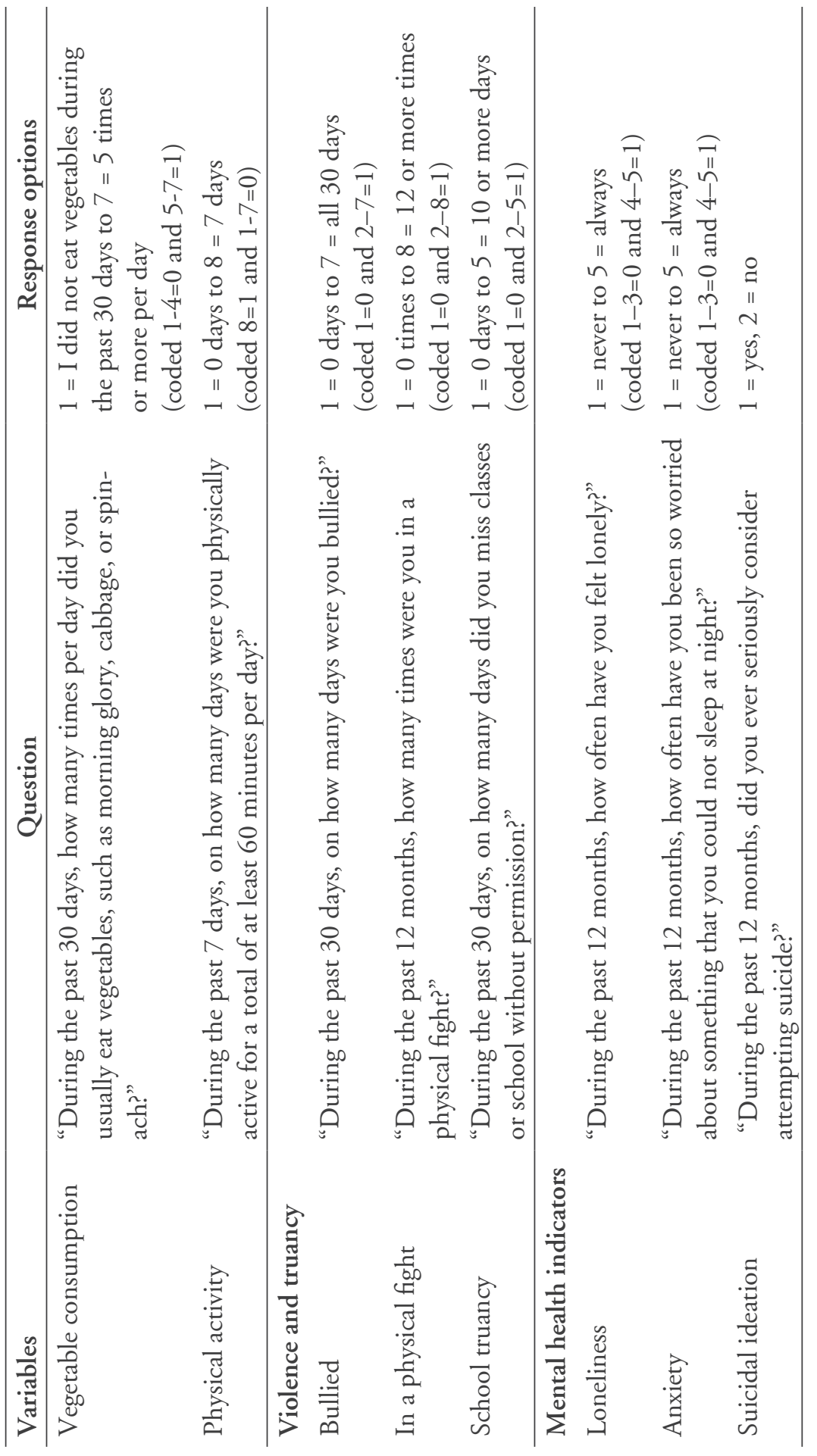




\section{Data analysis}

Descriptive statistics were used to describe the data, Chi-square statistics were used to test for differences in proportion, and one-way ANOVA was used to test for differences in means. The dichotomized responses of the four parental involvement items were added up to create a scale from 0 to 4 , with 4 reflecting the highest level of parental involvement. Logistic regression was used for assessing the impact of parental involvement variables and nine different health behaviors for each country separately. The regression model was adjusted with variables that could influence parental involvement, i.e., age, gender, socioeconomic status or hunger, and peer support. Both the 95\% confidence intervals and P-values were adjusted for the multi-stage, stratified, cluster sampling strategy of the study. All analyses were done with STATA software version 12.0 (Stata Corporation, College Station, TX, USA).

\section{RESULTS}

\section{Sample characteristics}

The sample included 44,231 schoolgoing adolescents (mean age 15.1 years, $\mathrm{SD}=1.5$ ) from Iraq (response rate $88 \%$ ), Kuwait (85\%), Malaysia (89\%), Mongolia (88\%), Philippines (82\%), and Vietnam (96\%). The number of students participating in the study in each country ranged from 2,072 in Kuwait to 25,507 in Malaysia.

While roughly a quarter to a third of students reported mostly or always receiving parental or guardian supervision, connectedness, or bonding, two thirds reported to be never or rarely monitored by their parents or guardians. Approximately four in ten students ate less than two servings of fruit per day. Only one in eight met the physical activity guidelines of at least 60 minutes per day, 7 days a week. Approximately a quarter to a third of all students conceded that they had been bullied in the past month, had been in a physical fight in the past 12 months, or had engaged in school truancy in the past month. In the past 12 months, one in eight students felt mostly or always lonely, one in ten had mostly or always experienced anxiety or worry, and one in six had seriously considered committing suicide (Table 2). 


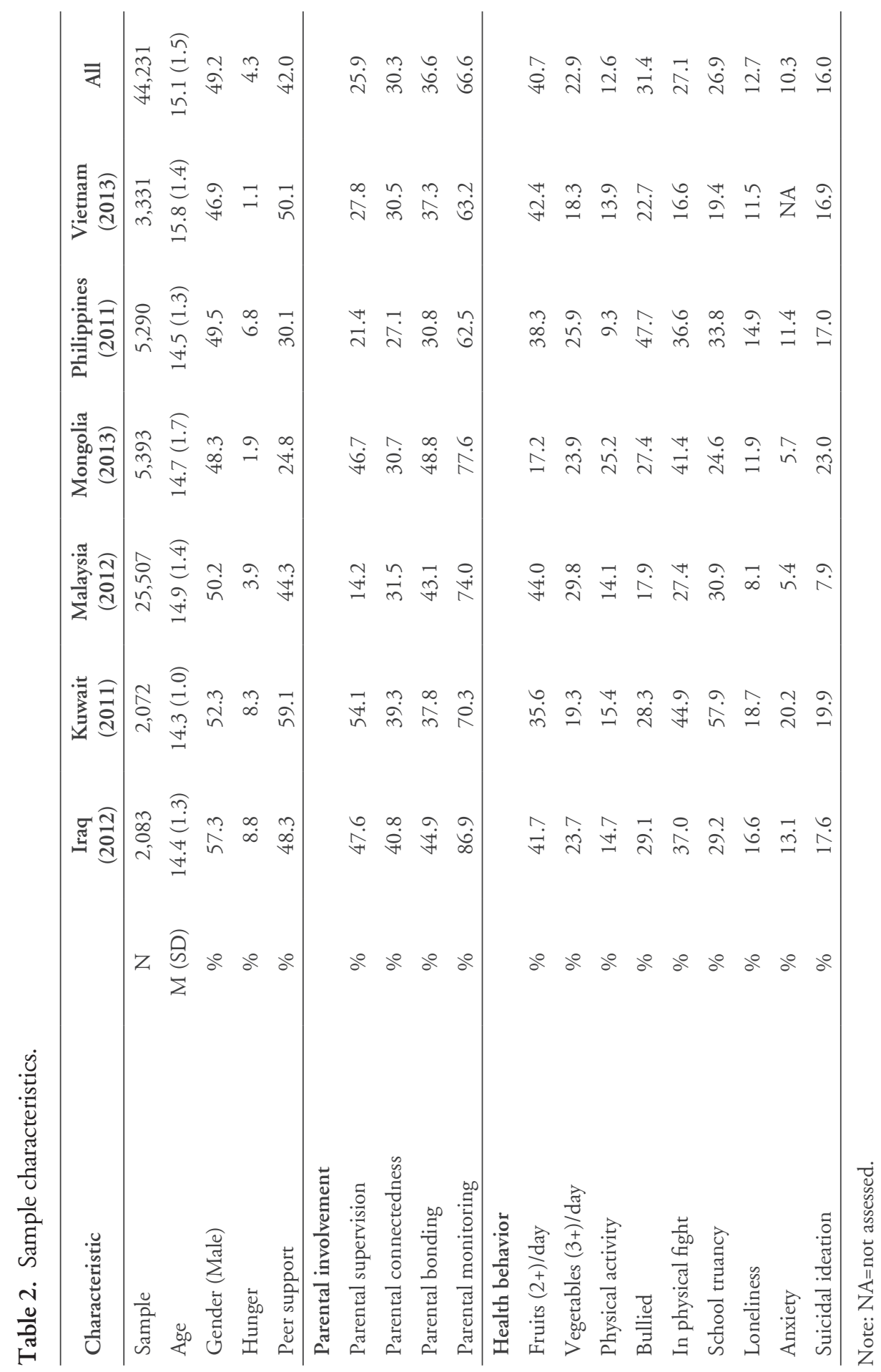


Bivariate analysis of parental involvement

More than one half of the students reported none to only one level of parental involvement; only $6 \%$ reported four levels of parental involvement. High parental involvement was associated with younger age, less experience of hunger and peer support, but did not differ in terms of gender.
Fruit consumption increased in all countries, except for Kuwait, as parental involvement increased. In contrast, vegetable consumption increased in three countries and did not increase in three countries as parental involvement increased. Physical activity increased in five countries, except for the Philippines, as parental involvement increased (Tables 3 and 4).

Table 3. Bivariate analysis with sociodemographic variables according to the level of parental involvement.

\begin{tabular}{|c|c|c|c|c|c|c|}
\hline \multirow{3}{*}{ Demographic } & \multicolumn{5}{|c|}{ Level of parental involvement } & \multirow{3}{*}{$\begin{array}{l}\text { Statistic } \\
\text { P-value }\end{array}$} \\
\hline & 0 & 1 & 2 & 3 & 4 & \\
\hline & $\%$ & $\%$ & $\%$ & $\%$ & $\%$ & \\
\hline n $(\%)$ & $\begin{array}{l}3,824 \\
(13.3)\end{array}$ & $\begin{array}{c}17,497 \\
(41.7)\end{array}$ & $\begin{array}{l}11,665 \\
(23.2)\end{array}$ & $\begin{array}{l}7,431 \\
(15.6)\end{array}$ & $\begin{array}{c}2,864 \\
(6.2)\end{array}$ & \\
\hline Age in years; $M(S D)$ & $\begin{array}{l}15.2 \\
(1.5)\end{array}$ & $\begin{array}{l}15.1 \\
(1.5)\end{array}$ & $\begin{array}{l}15.0 \\
(1.5)\end{array}$ & $\begin{array}{l}14.8 \\
(1.5)\end{array}$ & $\begin{array}{l}14.7 \\
(1.5)\end{array}$ & $<0.000$ \\
\hline Gender (male) & 48.4 & 49.2 & 48.5 & 49.4 & 48.7 & 0.089 \\
\hline Hunger & 4.1 & 5.1 & 4.1 & 3.2 & 1.9 & $<0.001$ \\
\hline Peer support & 33.2 & 30.9 & 47.0 & 61.3 & 70.7 & $<0.001$ \\
\hline
\end{tabular}

Table 4. Bivariate analysis with diet and physical activity according to the level of parental involvement.

\begin{tabular}{lcccccc}
\hline & \multicolumn{7}{c}{ Level of parental involvement } & Statistic \\
\cline { 2 - 6 } Health behavior & $\mathbf{0}$ & $\mathbf{1}$ & $\mathbf{2}$ & $\mathbf{3}$ & $\mathbf{4}$ & $\begin{array}{c}\text { P-value } \\
\%\end{array}$ \\
\cline { 2 - 6 } & $\%$ & $\%$ & $\%$ & $\%$ & \\
\hline Fruits 2 or more/day & & & & & & \\
Iraq & 44.3 & 37.1 & 38.9 & 44.6 & 50.5 & 0.002 \\
Kuwait & 28.4 & 35.2 & 35.7 & 33.3 & 42.7 & 0.256 \\
Malaysia & 43.4 & 41.1 & 43.8 & 48.7 & 60.3 & $<0.001$ \\
Mongolia & 11.5 & 14.7 & 16.2 & 18.5 & 23.6 & $<0.001$ \\
Philippines & 37.0 & 36.5 & 38.0 & 44.8 & 46.0 & 0.023 \\
Vietnam & 41.3 & 38.4 & 41.3 & 49.8 & 53.8 & $<0.001$ \\
\hline
\end{tabular}




\begin{tabular}{lrrrrrr}
\hline & \multicolumn{7}{c}{ Level of parental involvement } & Statistic \\
\cline { 2 - 5 } Health behavior & 0 & 1 & 2 & 3 & 4 & P-value \\
& $\%$ & $\%$ & $\%$ & $\%$ & $\%$ & \\
\hline Vegetables 3 or more/day & & & & & & \\
Iraq & 27.5 & 23.7 & 22.2 & 22.0 & 24.7 & 0.693 \\
Kuwait & 19.2 & 17.2 & 19.1 & 17.9 & 25.0 & 0.339 \\
Malaysia & 28.9 & 26.7 & 30.0 & 35.1 & 43.2 & $<0.001$ \\
Mongolia & 19.1 & 19.1 & 22.8 & 28.5 & 31.8 & $<0.001$ \\
Philippines & 25.1 & 26.0 & 24.5 & 28.8 & 26.9 & 0.450 \\
Vietnam & 17.0 & 16.1 & 19.0 & 20.2 & 28.1 & 0.004 \\
\hline Physical activity & & & & & & \\
Iraq & 10.1 & 9.7 & 15.4 & 18.7 & 19.8 & $<0.001$ \\
Kuwait & 14.3 & 10.0 & 17.9 & 16.1 & 20.3 & 0.038 \\
Malaysia & 14.1 & 11.4 & 14.6 & 18.8 & 24.5 & $<0.001$ \\
Mongolia & 19.0 & 19.8 & 24.1 & 29.9 & 36.0 & $<0.001$ \\
Philippines & 6.9 & 8.6 & 11.3 & 12.1 & 13.6 & 0.060 \\
Vietnam & 15.1 & 11.1 & 14.9 & 14.0 & 22.7 & 0.004 \\
\hline
\end{tabular}

Violent or aggressive behavior increased. In all but one country, (being bullied and having been in loneliness and anxiety decreased a physical fight) decreased in all six as parental involvement increased, countries as parental involvement and in all countries suicidal ideation increased. Likewise, school truancy decreased as parental involvement decreased in all countries, except increased (Table 5). for Kuwait, as parental involvement

Table 5. Bivariate analysis with violence, truancy, and mental health variables according to the level of parental involvement.

\begin{tabular}{lcccccc}
\hline & \multicolumn{7}{c}{ Level of parental involvement } & Statistic \\
\cline { 2 - 6 } Health behavior & $\mathbf{0}$ & $\mathbf{1}$ & $\mathbf{2}$ & 3 & 4 & P-value \\
& $\%$ & $\%$ & $\%$ & $\%$ & $\%$ & \\
\hline Bullied & 52.2 & 33.9 & 30.2 & 24.4 & 21.0 & $<0.001$ \\
Iraq & 45.7 & 30.3 & 30.7 & 25.0 & 15.1 & $<0.001$ \\
Kuwait & 24.1 & 19.6 & 16.8 & 12.7 & 11.8 & $<0.001$ \\
Malaysia & 39.2 & 29.7 & 27.0 & 26.4 & 18.6 & $<0.001$ \\
Mongolia & 54.2 & 49.3 & 45.0 & 40.4 & 47.5 & $<0.001$ \\
Philippines & 29.7 & 24.4 & 21.1 & 16.1 & 16.5 & $<0.001$ \\
Vietnam & & & & & &
\end{tabular}




\begin{tabular}{|c|c|c|c|c|c|c|}
\hline \multirow{3}{*}{ Health behavior } & \multicolumn{5}{|c|}{ Level of parental involvement } & \multirow{3}{*}{$\begin{array}{l}\text { Statistic } \\
\text { P-value }\end{array}$} \\
\hline & 0 & 1 & 2 & 3 & 4 & \\
\hline & $\%$ & $\%$ & $\%$ & $\%$ & $\%$ & \\
\hline \multicolumn{7}{|l|}{ In physical fight } \\
\hline Iraq & 43.4 & 39.1 & 39.3 & 34.2 & 29.8 & 0.003 \\
\hline Kuwait & 54.9 & 43.7 & 43.3 & 47.7 & 36.9 & 0.016 \\
\hline Malaysia & 39.4 & 29.4 & 24.5 & 21.2 & 21.2 & $<0.001$ \\
\hline Mongolia & 45.9 & 41.8 & 43.7 & 40.2 & 35.6 & 0.011 \\
\hline Philippines & 42.5 & 39.7 & 33.3 & 27.5 & 22.5 & $<0.001$ \\
\hline Vietnam & 22.0 & 17.3 & 14.5 & 14.6 & 8.4 & $<0.001$ \\
\hline \multicolumn{7}{|l|}{ School truancy } \\
\hline Iraq & 35.6 & 37.7 & 28.7 & 24.3 & 17.2 & $<0.001$ \\
\hline Kuwait & 63.2 & 59.9 & 59.8 & 56.7 & 51.1 & 0.161 \\
\hline Malaysia & 40.9 & 34.3 & 27.8 & 23.5 & 20.6 & $<0.001$ \\
\hline Mongolia & 37.3 & 28.2 & 25.1 & 20.1 & 15.2 & $<0.001$ \\
\hline Philippines & 38.4 & 34.4 & 32.7 & 29.8 & 19.5 & 0.003 \\
\hline Vietnam & 25.7 & 21.0 & 19.5 & 14.5 & 6.7 & $<0.001$ \\
\hline \multicolumn{7}{|l|}{ Loneliness } \\
\hline Iraq & 26.7 & 21.5 & 17.4 & 13.2 & 9.4 & $<0.001$ \\
\hline Kuwait & 31.7 & 26.0 & 16.6 & 13.5 & 7.0 & $<0.001$ \\
\hline Malaysia & 10.2 & 9.2 & 7.2 & 5.5 & 5.2 & $<0.001$ \\
\hline Mongolia & 23.5 & 13.7 & 11.1 & 9.8 & 6.8 & $<0.001$ \\
\hline Philippines & 15.7 & 16.2 & 13.6 & 12.2 & 10.9 & 0.137 \\
\hline Vietnam & 18.6 & 12.3 & 10.4 & 6.5 & 4.4 & $<0.001$ \\
\hline \multicolumn{7}{|l|}{ Anxiety } \\
\hline Iraq & 22.4 & 16.9 & 13.2 & 11.7 & 6.4 & $<0.001$ \\
\hline Kuwait & 29.7 & 24.3 & 20.0 & 17.5 & 10.6 & $<0.001$ \\
\hline Malaysia & 6.5 & 5.8 & 5.2 & 4.2 & 3.3 & 0.003 \\
\hline Mongolia & 12.8 & 7.2 & 4.6 & 3.8 & 3.5 & $<0.001$ \\
\hline Philippines & 11.7 & 11.3 & 11.4 & 12.8 & 10.6 & 0.894 \\
\hline Vietnam & NA & & & & & \\
\hline \multicolumn{7}{|l|}{ Suicidal ideation } \\
\hline Iraq & 37.4 & 21.6 & 17.4 & 15.3 & 8.5 & $<0.001$ \\
\hline Kuwait & 47.6 & 24.9 & 16.5 & 14.2 & 5.5 & $<0.001$ \\
\hline Malaysia & 13.4 & 9.5 & 6.0 & 5.0 & 3.1 & $<0.001$ \\
\hline Mongolia & 43.6 & 28.9 & 21.5 & 16.7 & 10.3 & $<0.001$ \\
\hline Philippines & 22.9 & 18.6 & 15.8 & 10.4 & 6.7 & $<0.001$ \\
\hline Vietnam & 30.8 & 19.4 & 12.2 & 7.6 & 7.2 & $<0.001$ \\
\hline
\end{tabular}


Association between level of parental involvement and health behaviors

In multivariable regression analyses, adjusted for age, gender, socioeconomic status, and peer support, higher levels of parental involvement were positively associated with fruit and vegetable consumption in Malaysia, Mongolia, and Vietnam, and with physical activity in Malaysia and Mongolia (Table 6).

Table 6. Multivariable logistic regression analyses of the level of parental involvement (presented as odds ratio with 95\% confidence interval; reference category: no parental involvement) on dietary behavior and physical activity.

\begin{tabular}{|c|c|c|c|c|}
\hline \multirow{2}{*}{ Country } & \multirow{2}{*}{$\begin{array}{c}\text { Level of } \\
\text { parental } \\
\text { involve- } \\
\text { ment }^{1}\end{array}$} & \multicolumn{3}{|c|}{ Dietary behavior and physical activity } \\
\hline & & Fruit & Vegetables & Physical activity \\
\hline \multirow[t]{4}{*}{ Iraq } & Level 1 & $0.76(0.40,1.43)$ & $0.82(0.48,1.38)$ & $0.96(0.36,2.61)$ \\
\hline & Level 2 & $0.78(0.44,1.40)$ & $0.79(0.45,1.37)$ & $1.52(0.57,4.06)$ \\
\hline & Level 3 & $0.97(0.52,1.80)$ & $0.73(0.44,1.21)$ & $1.76(0.68,4.57)$ \\
\hline & Level 4 & $1.20(0.69,2.09)$ & $0.85(0.44,1.65)$ & $1.66(0.61,4.53)$ \\
\hline \multirow[t]{4}{*}{ Kuwait } & Level 1 & $1.31(0.64,2.67)$ & $0.82(0.34,1.98)$ & $0.58(0.25,1.36)$ \\
\hline & Level 2 & $1.34(0.63,2.85)$ & $0.93(0.37,2.35)$ & $1.17(0.44,3.09)$ \\
\hline & Level 3 & $1.13(0.52,2.50)$ & $0.80(0.27,2.35)$ & $0.81(0.35,1.87)$ \\
\hline & Level 4 & $1.60(0.70,3.64)$ & $1.18(0.39,3.64)$ & $1.10(0.40,3.00)$ \\
\hline \multirow[t]{4}{*}{ Malaysia } & Level 1 & $0.92(0.82,1.03)$ & $0.90(0.78,1.02)$ & $0.80(0.65,0.99)^{*}$ \\
\hline & Level 2 & $1.00(0.90,1.11)$ & $1.03(0.90,1.19)$ & $1.00(0.82,1.23)$ \\
\hline & Level 3 & $1.18(1.03,1.35)^{*}$ & $1.27(1.10,1.47)^{* *}$ & $1.32(1.09,1.59)^{* *}$ \\
\hline & Level 4 & $1.77(1.41,2.21)^{* * *}$ & $1.74(1.47,2.08)^{* * *}$ & $1.85(1.45,2.37)^{* * *}$ \\
\hline \multirow[t]{4}{*}{ Mongolia } & Level 1 & $1.29(0.93,1.78)$ & $0.97(0.73,1.30)$ & $1.03(0.77,1.37)$ \\
\hline & Level 2 & $1.38(0.91,2.09)$ & $1.14(0.86,1.53)$ & $1.27(0.94,1.72)$ \\
\hline & Level 3 & $1.56(1.09,2.25)^{*}$ & $1.42(1.10,1.85)^{* *}$ & $1.66(1.23,2.24)^{* * *}$ \\
\hline & Level 4 & $2.01(1.40,2.91)^{* * *}$ & $1.56(1.16,2.08)^{* *}$ & $2.15(1.57,2.95)^{* * *}$ \\
\hline \multirow[t]{4}{*}{ Philippines } & Level 1 & $0.99(0.83,1.19)$ & $1.03(0.79,1.32)$ & $1.28(0.80,2.05)$ \\
\hline & Level 2 & $1.06(0.85,1.34)$ & $0.93(0.67,1.27)$ & $1.58(1.12,2.23)^{*}$ \\
\hline & Level 3 & $1.43(1.12,1.81)^{* *}$ & $1.16(0.85,1.57)$ & $1.46(0.97,2.21)$ \\
\hline & Level 4 & $1.48(0.96,2.27)$ & $1.04(0.66 .1 .65)$ & $1.64(0.63,4.26)$ \\
\hline \multirow[t]{4}{*}{ Vietnam } & Level 1 & $0.90(0.71,1.15)$ & $0.93(0.65,1.33)$ & $0.69(0.47,1.01)$ \\
\hline & Level 2 & $0.99(0.74,1.33)$ & $1.10(0.75,1.62)$ & $0.93(0.57,1.54)$ \\
\hline & Level 3 & $1.33(0.99,1.78)$ & $1.10(0.77,1.57)$ & $0.84(0.58,1.21)$ \\
\hline & Level 4 & $1.58(1.08,2.33)^{*}$ & $1.69(1.06,2.70)^{*}$ & $1.55(0.88,2.75)$ \\
\hline
\end{tabular}

Note: ${ }^{1}$ Adjusted for age, gender, socioeconomic status (experience of hunger), and peer support. ${ }^{* * *} \mathrm{P}<0.001 ;{ }^{* *} \mathrm{P}<0.01 ;{ }^{*} \mathrm{P}<0.05$. 
Further, higher levels of parental been in a physical fight in all six involvement were negatively associated countries, and with school truancy with bullying victimization and having in five countries (Table 7).

Table 7. Multivariable logistic regression analyses of the level of parental involvement (presented as odds ratio with 95\% confidence interval; reference category: no parental involvement) on violence and truancy.

\begin{tabular}{|c|c|c|c|c|}
\hline \multirow[b]{2}{*}{ Country } & \multirow{2}{*}{$\begin{array}{l}\text { Level of } \\
\text { parental } \\
\text { involve- } \\
\text { ment }^{1}\end{array}$} & \multicolumn{3}{|c|}{ Violence and truancy } \\
\hline & & $\begin{array}{c}\text { Bullying } \\
\text { victimization }\end{array}$ & In physical fight & School truancy \\
\hline \multirow[t]{4}{*}{ Iraq } & Level 1 & $0.50(0.31,0.81)^{* *}$ & $0.90(0.54,1.50)$ & $1.03(0.53,2.01)$ \\
\hline & Level 2 & $0.48(0.27,0.85)^{*}$ & $0.98(0.59,1.65)$ & $0.75(0.39,1.42)$ \\
\hline & Level 3 & $0.37(0.20,0.67)^{* *}$ & $0.74(0.43,1.28)$ & $0.63(0.33,1.21)$ \\
\hline & Level 4 & $0.30(0.18,0.50)^{* * *}$ & $0.58(0.35,0.97)^{*}$ & $0.43(0.20,0.90)^{*}$ \\
\hline \multirow[t]{4}{*}{ Kuwait } & Level 1 & $0.48(0.30,0.76)^{* *}$ & $0.54(0.36,0.81)^{* *}$ & $0.94(0.57,1.56)$ \\
\hline & Level 2 & $0.53(0.29,0.98)^{*}$ & $0.57(0.38,0.85)^{* *}$ & $0.89(0.49,1.61)$ \\
\hline & Level 3 & $0.34(0.17,0.69)^{* *}$ & $0.58(0.36,0.95)^{*}$ & $0.81(0.47,1.39)$ \\
\hline & Level 4 & $0.18(0.09,0.39)^{* * *}$ & $0.36(0.21,0.62)^{* * *}$ & $0.61(0.35,1.07)$ \\
\hline \multirow[t]{4}{*}{ Malaysia } & Level 1 & $0.81(0.67,0.99)^{*}$ & $0.67(0.57,0.78)^{* * *}$ & $0.75(0.65,0.85)^{* * *}$ \\
\hline & Level 2 & $0.71(0.60,0.85)^{* * *}$ & $0.54(0.45,0.64)^{* * *}$ & $0.58(0.50,0.66)^{* * *}$ \\
\hline & Level 3 & $0.54(0.43,0.67)^{* * *}$ & $0.46(0.39,0.55)^{* * *}$ & $0.49(0.43,0.56)^{* * *}$ \\
\hline & Level 4 & $0.47(0.34,0.65)^{* * *}$ & $0.46(0.36,0.60)^{* * *}$ & $0.46(0.36,0.57)^{* * *}$ \\
\hline \multirow[t]{4}{*}{ Mongolia } & Level 1 & $0.64(0.48,0.85)^{* *}$ & $0.76(0.63,0.92)^{* *}$ & $0.63(0.50,0.80)^{* * *}$ \\
\hline & Level 2 & $0.53(0.39,0.73)^{* * *}$ & $0.78(0.61,1.00)$ & $0.54(0.43,0.69)^{* * *}$ \\
\hline & Level 3 & $0.50(0.38,0.67)^{* * *}$ & $0.70(0.55,0.89)^{* *}$ & $0.45(0.36,0.57)^{* * *}$ \\
\hline & Level 4 & $0.33(0.23,0.47)^{* * *}$ & $0.57(0.44,0.76)^{* * *}$ & $0.35(0.25,0.48)^{* * *}$ \\
\hline \multirow[t]{4}{*}{ Philippines } & Level 1 & $0.83(0.66,1.04)$ & $0.90(0.73,1.12)$ & $0.78(0.65,0.94)^{* *}$ \\
\hline & Level 2 & $0.73(0.56,0.96)^{*}$ & $0.72(0.58,0.90)^{* *}$ & $0.79(0.63,0.98)^{*}$ \\
\hline & Level 3 & $0.61(0.43,0.86)^{* *}$ & $0.57(0.41,0.79)^{* * *}$ & $0.77(0.57,1.04)$ \\
\hline & Level 4 & $0.83(0.54,1.27)$ & $0.44(0.30,0.66)^{* * *}$ & $0.44(0.26,0.77)^{* *}$ \\
\hline \multirow[t]{4}{*}{ Vietnam } & Level 1 & $0.76(0.59,0.97)^{*}$ & $0.73(0.56,0.97)^{*}$ & $0.75(0.58,0.98)^{*}$ \\
\hline & Level 2 & $0.65(0.48,0.88)^{* *}$ & $0.58(0.43,0.77)^{* * *}$ & $0.71(0.53,0.94)^{*}$ \\
\hline & Level 3 & $0.47(0.34,0.66)^{* * *}$ & $0.50(0.36,0.71)^{* * *}$ & $0.52(0.39,0.75)^{* * *}$ \\
\hline & Level 4 & $0.53(0.32,0.88)^{*}$ & $0.29(0.17,0.49)^{* * *}$ & $0.23(0.12,0.46)^{* * *}$ \\
\hline
\end{tabular}

Note: ${ }^{1}$ Adjusted for age, gender, socioeconomic status (experience of hunger), and peer support. ${ }^{* * *} \mathrm{P}<0.001 ;{ }^{* *} \mathrm{P}<0.01 ;{ }^{*} \mathrm{P}<0.05$. 
Regarding mental health, higher ideation in all six countries, loneliness levels of parental involvement were in five countries, and anxiety in four negatively associated with suicidal countries (Table 8).

Table 8. Multivariable logistic regression analyses of the level of parental involvement (presented as odds ratio with 95\% confidence interval; reference category: no parental involvement) on mental health indicators.

\begin{tabular}{|c|c|c|c|c|}
\hline \multirow{2}{*}{ Country } & \multirow{2}{*}{$\begin{array}{c}\text { Level of } \\
\text { parental } \\
\text { involvement }\end{array}$} & \multicolumn{3}{|c|}{ Mental health } \\
\hline & & Loneliness & Anxiety & Suicidal ideation \\
\hline \multirow[t]{4}{*}{ Iraq } & Level 1 & $0.66(0.33,1.31)$ & $0.53(0.25,1.12)$ & $0.39(0.18,0.82)^{*}$ \\
\hline & Level 2 & $0.52(0.24,1.11)$ & $0.44(0.20,0.94)^{*}$ & $0.33(0.15,0.70)^{* *}$ \\
\hline & Level 3 & $0.41(0.20,0.87)^{*}$ & $0.42(0.19,0.91)^{*}$ & $0.32(0.15,0.68)^{* *}$ \\
\hline & Level 4 & $0.29(0.12,0.73)^{*}$ & $0.23(0.11,0.48)^{* * *}$ & $0.18(0.08,0.40)^{* * *}$ \\
\hline \multirow[t]{4}{*}{ Kuwait } & Level 1 & $0.83(0.59,1.14)$ & $0.81(0.55,1.19)$ & $0.37(0.23,0.59)^{* * *}$ \\
\hline & Level 2 & $0.47(0.33,0.68)^{* * *}$ & $0.64(0.45,0.92)^{*}$ & $0.23(0.14,0.38)^{* * *}$ \\
\hline & Level 3 & $0.42(0.29,0.60)^{* * *}$ & $0.59(0.39,0.91)^{*}$ & $0.20(0.09,0.41)^{* * *}$ \\
\hline & Level 4 & $0.21(0.11,0.42)^{* * *}$ & $0.35(0.18,0.66)^{* *}$ & $0.06(0.03,0.14)^{* * *}$ \\
\hline \multirow[t]{4}{*}{ Malaysia } & Level 1 & $0.88(0.68,1.13)$ & $0.88(0.66,1.16)$ & $0.68(0.56,0.82)^{* * *}$ \\
\hline & Level 2 & $0.68(0.54,0.85)^{* * *}$ & $0.78(0.55,1.10)$ & $0.43(0.34,0.55)^{* * *}$ \\
\hline & Level 3 & $0.50(0.39,0.65)^{* * *}$ & $0.64(0.46,0.86)^{* *}$ & $0.37(0.29,0.47)^{* * *}$ \\
\hline & Level 4 & $0.51(0.34,0.75)^{* * *}$ & $0.53(0.32,0.88)^{*}$ & $0.23(0.15,0.36)^{* * *}$ \\
\hline \multirow[t]{4}{*}{ Mongolia } & Level 1 & $0.62(0.40,0.70)^{* * *}$ & $0.50(0.34,0.72)^{* * *}$ & $0.53(0.41,0.69)^{* * *}$ \\
\hline & Level 2 & $0.42(0.31,0.58)^{* * *}$ & $0.33(0.20,0.55)^{* * *}$ & $0.39(0.30,0.48)^{* * *}$ \\
\hline & Level 3 & $0.36(0.27,0.47)^{* * *}$ & $0.28(0.18,0.43)^{* * *}$ & $0.27(0.21,0.36)^{* * *}$ \\
\hline & Level 4 & $0.24(0.16,0.37)^{* * *}$ & $0.26(0.16,0.45)^{* * *}$ & $0.16(0.12,0.22)^{* * *}$ \\
\hline \multirow[t]{4}{*}{ Philippines } & Level 1 & $1.02(0.77,1.39)$ & $0.95(0.70,1.29)$ & $0.74(0.59,0.93)^{*}$ \\
\hline & Level 2 & $0.87(0.64,1.19)$ & $0.95(0.62,1.47)$ & $0.64(0.49,0.83)^{* * *}$ \\
\hline & Level 3 & $0.79(0.51,1.23)$ & $0.99(0.64,1.52)$ & $0.43(0.29,0.62)^{* * *}$ \\
\hline & Level 4 & $0.71(0.46,1.09)$ & $0.79(0.38,1.65)$ & $0.27(0.13,0.56)^{* * *}$ \\
\hline \multirow[t]{4}{*}{ Vietnam } & Level 1 & $0.60(0.45,0.81)^{* * *}$ & NA & $0.52(0.40,0.67)^{* * *}$ \\
\hline & Level 2 & $0.53(0.38,0.73)^{* * *}$ & & $0.31(0.20,0.46)^{* * *}$ \\
\hline & Level 3 & $0.34(0.23,0.50)^{* * *}$ & & $0.20(0.13,0.31)^{* * *}$ \\
\hline & Level 4 & $0.23(0.12,0.45)^{* * *}$ & & $0.19(0.10,0.36)^{* * *}$ \\
\hline
\end{tabular}

Note: NA=not assessed. ${ }^{1}$ Adjusted for age, gender, socioeconomic status (experience of hunger), and peer support. ${ }^{* *} \mathrm{P}<0.001 ;{ }^{* *} \mathrm{P}<0.01 ;{ }^{*} \mathrm{P}<0.05$. 


\section{DISCUSSION}

This study of school-going adolescents in six Asian countries from different regions (Middle East, Central, and Southeast Asia) agreed with previous studies (Heitzler et al., 2006; Raudsepp, 2006; Mistry et al., 2009; Pearson et al., 2010; Cutler et al., 2011; Yao \& Rhodes, 2015; and Ahmed et al., 2016) that higher levels of parental involvement were positively associated with fruit and vegetable consumption and physical activity in half of the study countries (Malaysia, Mongolia, and Vietnam). One possible reason for the mixed results of the relationship between parental involvement and dietary and physical activity behavior in Iraq, Kuwait, and Philippines may be that the extended family played a larger role in child care activities in these countries, and this was not measured in our study (Arat and Wong, 2016). In addition, parental involvement was the lowest among the six countries in the Philippines, which have may contributed to the non-significant results regarding dietary and physical activity behavior there. In a UNICEF report, among other countries, the Philippines also ranked poorly in child well-being in the Pacific region (Daly et al., 2015).

Consistent with previous studies (Siziya et al., 2007; Hazemba et al., 2008; Rudatsikira et al., 2008; Hoeve, 2009; Muula et al., 2012; and Erginoz, 2015), this study also found a negative association between parental involvement and violence (being bullied and in a physical fight) and truancy. An intervention trial that utilized parental involvement reduced truant and other problem behaviors among adolescents (Stanton et al., 2004). Likewise, parental involvement and support can be utilized in interventions targeted to reduce aggressive and truancy behaviors in adolescents.

Regarding mental health, in almost all countrires, increasing parental involvement prevented internalizing problems, including loneliness, anxiety, and suicidal ideation. These findings are consistent with a number of previous studies and reviews (Barber, 2002; Abdirahman et al., 2012; Hasumi et al., 2012; Tammariello et al., 2012; Yap et al., 2016; Arat \& Wong, 2016; and Wu \& Yaacob, 2017;).

Our study findings also showed that, generally, only one level of parental involvement had a protective effect in most countries on being bullied, in a physical fight, school truancy, and suicidal ideation, while three or four levels were needed for fruit and vegetable consumption and physical activity. The effect seems to become stronger with increasing levels of parental involvement, and could possibly be used by parents or guardians to improve on the prevalence of various health behaviors of their adolescent children. Parenting support, in the form of health-promoting interventions for parents and children and general support for parents, could be strengthened in the study countries (Daly et al., 2015), to eventually improve health promotion targets. 


\section{Study limitations}

Due to the cross-sectional study design, causal inferences cannot be made. The study included only adolescents who were attending school and those not attending or out of school were not included in the study, so generalizations cannot be made for the total adolescent population in each country. Further, the self-report of certain health behaviors, such as having been in a physical fight, should be interpreted with caution, because of poor memory recall and possibly underreporting. Moreover, several indicators were only measured with a single item, which has its limitations. Some of the variables assessed in the GSHS were not available for all the study countries. The study only assessed generic parental involvement, and did not assess parental involvement related to specific health behaviors, such as fruit and vegetable consumption or physical activity, which should be assessed in future studies.

\section{CONCLUSION}

The study found that a considerable proportion of adolescents in six Asian countries engage in various health risk behaviors. Parental involvement and/or support may be effective in increasing the number of healthy behaviors, including fruit and vegetable consumption, physical activity, no violent behaviors, school attendance, and mental health in this adolescent population. The magnitude of the possible influence of parental involvement on adolescent health behaviors, including mental health, necessitates more research, social policy, and implementation on parental or familybased interventions for adolescent health promotion in the Asian region.

\section{ACKNOWLEDGEMENTS}

We are grateful to the World Health Organization (Geneva) for making the data available to us for analysis. We also thank the Ministries of Education and Health and the study participants for making the Global School Health Survey possible in the study countries. The governments of the study countries and the World Health Organization did not influence the analysis, nor did they influence the decision to publish these findings (CDC, 2017).

\section{REFERENCES}

Abdirahman, H. A., Bah, T. T., Shrestha, H.L., \& Jacobsen, K.H. (2012). Bullying, mental health, and parental involvement among adolescents in the Caribbean. West Indian Medical Journal, 61(5), 504-508.

Ahmed, J., Mehraj, V., Jeswani, G.K., Rehman, S., Shah, S.M., \& Hamadeh, R. (2016). Parental and school influences on physical activity levels of high school students in Hyderabad, Pakistan. Journal of Ayub Medical College, Abbottabad, 28(1), 110-115. 
Arat, G. \& Wong, P.W.C. (2016). The relationship between parental involvement and adolescent mental health in six sus-Saharan African countries: findings from Global School-based Health Surveys (GSHS). International Journal of Mental Health Promotion, 18(3), 144-157. https://doi.org/10.1080/ 14623730.2016.1194305

Barber, B.K. (2002). Regulation, connection, and psychological autonomy: Evidence from the Cross National Adolescent Project $(C-N A P)$. Paper presented at the WHO-sponsored meeting Regulation as a Concept and Construct for Adolescent Health and Development. WHO Headquarters, Geneva, Switzerland, April 16-18. Centers for Disease Control (CDC). (2013). State Indicator Report on Fruits and Vegetables. Retrieved from: http://www.cdc.gov/nutrition/ downloads/state-indicator-reportfruits-vegetables-2013.pdf

Centers for Disease Control and Prevention (CDC). (2017). Global School-based Student Health Survey (GSHS). Retrieved from https:// www.cdc.gov/gshs/

Cutler, G.J., Flood, A., Hannan, P., \& Neumark-Sztainer, D. (2011). Multiple sociodemographic and socioenvironmental characteristics are correlated with major patterns of dietary intake in adolescents. Journal of the American Dietetic Association, 111(2), 230-40. https:// doi.org/10.1016/j.jada.2010. 10.052
Daly, M., Bray, R., Bruckauf, Z., Byrne, J., Margaria, A., Pećnik, N., \& Samms-Vaughan, M. (2015). Family and Parenting Support: Policy and Provision in a Global Context, Innocenti Insight. Florence: UNICEF Office of Research.

de Looze, M., van den Eijnden, R., Verdurmen, J., Vermeulen-Smit, E., Schulten, I., Vollebergh. W., \& ter Bogt, T. (2012). Parenting practices and adolescent risk behavior: rules on smoking and drinking also predict cannabis use and early sexual debut. Prevention Science, 13(6), 594-604. ht tps://doi.org/10.1007/ s11121-012-0286-1

Erginoz, E., Alikasifoglu, M., Ercan, O., Uysal, O., Alp, Z., Ocak, S., ... Albayrak Kaymak, D. (2015). The role of parental, school, and peer factors in adolescent bullying involvement: results from the Turkish HBSC 2005/2006 study. Asia Pacific Journal of Public Health, 27(2), NP1591-603. https://doi.org/10. 1177/1010539512473144

Hasumi, T., Ahsan, F., Couper, C. M., Aguayo, J.L., \& Jacobsen, K.H. (2012). Parental involvement and mental well-being of Indian adolescents. Indian Pediatrics, 49(11), 915-918. 
Hazemba, A., Siziya, S., Muula, A.S., \& Rudatsikiram E. (2008). Prevalence and correlates of being bullied among in-school adolescents in Beijing: results from the 2003 Beijing Global School-Based Health Survey. Annals of General Psychiatry, 7, 6. https://doi.org/10.1186/1744859X-7-6

Heitzler, C.D., Martin, S.L., Duke, J., \& Huhman, M. (2006). Correlates of physical activity in a national sample of children aged 9-13 years. Preventive Medicine, 42(4), 254-260. https://doi.org/10.1016/j.ypmed. 2006.01.010

Hoeve, M., Dubas, J.S., Eichelsheim, V.I., van der Laan, P.H., Smeenk, W., \& Gerris, J.R. (2009). The relationship between parenting and delinquency: a meta-analysis. Journal of Abnormal Child Psychology, 37(6), 749-775. https:// doi.org/10.1007/s10802-0099310-8

Mistry, R., McCarthy, W.J., Yancey, A.K., Lu, Y., \& Patel, M. (2009). Resilience and patterns of health risk behaviors in California adolescents. Preventive Medicine, 48(3), 291-297. https://doi.org/ 10.1016/j.ypmed.2008.12.013

Muula, A.S., Rudatsikira, E., Babaniyi, O., Songolo, P., \& Siziya, S. (2012). Prevalence and correlates for school truancy among pupils in grades 7-10: results from the 2004 Zambia Global School-based Health Survey. BMC Research Notes, 5, 48. https://doi.org/10.1186/ 1756-0500-5-48
Newman, K., Harrison, L., Dashiff, C., \& Davies, S. (2008). Relationships between parenting styles and risk behaviors in adolescent health: an integrative literature review. Revista Latino-Americana de Enfermagem, 16(1):142-150.

Pearson, N., Atkin, A.J., Biddle, S.J., Gorely, T., \& Edwardson, C. (2010). Parenting styles, family structure and adolescent dietary behavior. Public Health Nutrition, 13(8), 1245-1253. https://doi.org/ $10.1017 /$ S1368980009992217

Raudsepp, L. (2006). The relationship between socio-economic status, parental support and adolescent physical activity. Acta Paediatrica, 95(1), 93-98.

Rudatsikira, E., Mataya, R.H., Siziya, S., \& Muulam A.S. (2008). Association between bullying victimization and physical fighting among Filipino adolescents: results from the Global School-Based Health Survey. Indian Journal of Pediatrics, 75(12), 1243-1247. https://doi.org/10.1007/s12098008-0244-x

Siziya, S., Muula, A.S., \& Rudatsikira, E. (2007). Prevalence and correlates of truancy among adolescents in Swaziland: findings from the Global School-based Health Survey. Child and Adolescent Psychiatry and Mental Health, 1, 15. https://doi.org/10.1186/17532000-1-15 
Stanton, B., Cole, M., Galbraith, J., Li, X., Pendleton, S., Cottrel, L.,...\& Kaljee, L. (2004). Randomized trial of a parent intervention: parents can make a difference in long-term adolescent risk behaviors, perceptions, and knowledge. Archives of Pediatrics \& Adolescent Medicine, 158, 947-955. https://doi.org/10.1001/archpedi. 158.10.947

Tammariello, A.E., Gallahue, N.K., Ellard, K.A., Woldesemait, N., \& Jacobson, K.H. (2012). Parental involvement and mental health among Thai adolescents. Advances in School Mental Health Promotion, 5(4), 236-245. https://doi.org/10. 1080/1754730X.2012.728095

Yao, C.A., \& Rhodes, R.E. (2015). Parental correlates in child and adolescent physical activity: a metaanalysis. International Journal of Behavioral Nutrition and Physical Activity, 12, 10. https://doi.org/ 10.1186/s12966-015-0163-y
Yap, M.B., Morgan, A.J., Cairns, K., Jorm, A.F., Hetrick, S.E., \& Merry, S. (2016). Parents in prevention: A meta-analysis of randomized controlled trials of parenting interventions to prevent internalizing problems in children from birth to age 18. Clinical Psychology Review, 50, 138-158. https://doi. org/10.1016/j.cpr.2016.10.003

World Health Organization (WHO) (2010). Global recommendations on physical activity for health. Geneva, Switzerland: WHO.

Wu, S.L., \& Yaacob, S.N. (2017). Self-efficacy as a mediator of the relationship between parental closeness and suicidal ideation among Malaysian adolescents. Child and Adolescent Mental Health, 12(10), 84-90. https://doi. org/10.1111/camh.12188 